\title{
Double open charm meson production at the LHC: new single- and double-parton scattering mechanisms
}

\author{
Rafał Maciuła $^{1, \star}$ and Antoni Szczurek ${ }^{1,2}$ \\ ${ }_{1}^{1}$ Institute of Nuclear Physics, Polish Academy of Sciences, Radzikowskiego 152, PL-31-342 Kraków, Poland \\ ${ }^{2}$ University of Rzeszów, PL-35-959 Rzeszów, Poland
}

\begin{abstract}
We discuss $D^{0} D^{0}$ meson-meson pair production related to the recent $\mathrm{LHCb}$ experimental results obtained at $\sqrt{s}=7 \mathrm{TeV}$. We consider double-parton scattering (DPS) mechanisms of double $c \bar{c}$ production and subsequent standard $c c \rightarrow D^{0} D^{0}$ scale-independent hadronization as well as double $g$ and mixed $g c \bar{c}$ production with $g g \rightarrow D^{0} D^{0}$ and $g c \rightarrow D^{0} D^{0}$ scale-dependent hadronization. The latter scenario with $g \rightarrow D^{0}$ fragmentation components results in a new single-parton scattering (SPS) mechanism of digluon production which is also taken here into account. Results of the numerical calculations are compared with the $\mathrm{LHCb}$ data for correlation observables in azimuthal angle $\varphi_{D^{0} D^{0}}$ between $D^{0}$ mesons, in dimeson invariant mass $M_{D^{0} D^{0}}$ and in transverse momentum of the meson from the $D^{0} D^{0}$ pair. The inclusion of the new mechanisms with $g \rightarrow D^{0}$ fragmentation leads to a larger cross sections and to slightly different shapes of the calculated correlation observables.
\end{abstract}

\section{Introduction}

Some time ago we have predicted that at large energies, relevant for the Large Hadron Collider, production of double charm should be dominated by the double-parton scattering (DPS) mechanism [1]. In the next step those predictions based on leading-order (LO) collinear approach were improved by application of the $k_{t}$-factorization approach which effectively includes higher-order QCD effects $[2,3]$. The extended studies provide a relatively good description of the LHCb experimental data [4] for both the total cross section and the dimeson correlation observables. Moreover, within these analyses the single-parton scattering (SPS) $g g \rightarrow c \bar{c} c \bar{c}$ contribution was also carefully discussed in both collinear [3] and $k_{t}$-factorization [5] approaches. The SPS contribution to the double $c \bar{c}$ cross section was found to be rather small, much smaller than the DPS one, and cannot explain the total and differential cross sections measured by the LHCb.

The studies introduced above were based on the standard $c \rightarrow D$ hadronization scenario with scale-independent Peterson fragmentation function (FF) [6]. An alternative approach for hadronization effects is to apply scale-dependent FFs of a parton (gluon, $u, d, s, \bar{u}, \bar{d}, \bar{s}, c, \bar{c}$ ) to $D$ mesons proposed by Kniehl et al. [7, 8], that undergo DGLAP evolution equations. Both prescriptions were found to provide a very good description of the LHC data on inclusive $D$ meson production (see e.g. Refs. $[9,10])$. In the latter approach, a dominant contribution comes from $g \rightarrow D$ fragmentation that

^ e-mail: rafal.maciula@ifj.edu.pl.pl, supported by the Polish National Science Center grant DEC-2014/15/B/ST2/02528 
appears in the evolution of the scale-dependent FFs and the $c \rightarrow D$ component is damped with respect to the scale-independent fragmentation scheme.

Here we wish to discuss how the presence of the gluonic components change the overall picture for the double $D$-meson production. In the scale-dependent (new) scenario for hadronization with $g \rightarrow D$ fragmentation the number of contributing DPS processes grows compared to the standard (old) scenario with $c \rightarrow D$ fragmentation only. Moreover, a new single-parton scattering mechanism SPS $g g \rightarrow D D$ appears. In the new scenario we have more processes for single $D$ meson production (two components) and as a consequence many more processes for the pair production in doubleparton scattering. Now there are three classes of DPS contributions. In addition to the coventional DPS $c c \rightarrow D D$, considered in Refs. [2, 3, 5] there is a double $g \rightarrow D$ fragmentation mechanism, called here DPS $g g \rightarrow D D$ as well as the mixed DPS $g c \rightarrow D D$ contribution.

Here the gluon and digluon production is considered in the $k_{t}$-factorization approach with Reggeized gluons in the t-channel [11] via subprocesses $R R \rightarrow g$ and $R R \rightarrow g g$, where $R$ is the Reggeized gluon. We use scale-dependent fragmentation functions of Kneesch-Kniehl-KramerSchienbein (KKKS08) [12] as implemented in the code available on the Web [13].

All details, relevant diagrams, and the theoretical formalism behind the calculations presented here can be found in our original paper [14] and is not repeated here. There, one can also find a revisited comparison of the theoretical predictions based on the two hadronization scenarios for single $D$ meson production, calculated with the same set of $\alpha_{S}$, scales, unPDFs and other details which are relevant for the discussion of double $D$ meson production.

\section{Comparison to the $\mathrm{LHCb}$ data}

We compare now results of our theoretical predictions for double charm production with the $\mathrm{LHCb}$ experimental data for $D^{0} D^{0}$ pair production. In Fig. 1 we compare results of our calculation with experimental distribution in transverse momentum of one of the meson from the $D^{0} D^{0}$ (or $\bar{D}^{0} \bar{D}^{0}$ ) pair. We show results for the first scenario when standard Peterson FF is used for the $c \rightarrow D^{0}$ (or $\bar{c} \rightarrow \bar{D}^{0}$ ) fragmentation (left panel) as well as the result for the second scenario when the KKKS08 FFs with DGLAP evolution for $c \rightarrow D^{0}$ (or $\bar{c} \rightarrow \bar{D}^{0}$ ) and $g \rightarrow D^{0}$ (or $g \rightarrow \bar{D}^{0}$ ) are used. One can observe that the DPS $c c \rightarrow D^{0} D^{0}$ contribution in the new scenario is much smaller than in the old scenario. In addition, the slope of the distribution in transverse momentum changes. Both the effects are due to evolution of corresponding fragmentation function in the second scenario, compared to lack of such an effect in the first scenario. The different new mechanisms give contributions of similar size. We can obtain an agreement in the second case provided $\sigma_{\text {eff }}$ parameter is increased from conventional $15 \mathrm{mb}$ to $30 \mathrm{mb}$. Even then we overestimate the $\mathrm{LHCb}$ data for $3<p_{T}<5 \mathrm{GeV}$. Possible effects that may result in larger value of $\sigma_{e f f}$ and in its transverse momentum dependence are discussed in our original paper [14].

In Fig. 2 we show dimeson invariant mass distribution $M_{D^{0} D^{0}}$ again for the two cases considered. In the first scenario we get a good agreement only for small invariant masses while in the second scenario we get a good agreement only for large invariant masses. The large invariant masses are strongly correlated with large transverse momenta, so the situation here (for the invariant mass distribution) is quite similar as in Fig. 1 for the transverse momentum distribution.

In Fig. 3 we show azimuthal angle correlation $\varphi_{D^{0} D^{0}}$ between $D^{0}$ and $D^{0}$ (or $\bar{D}^{0}$ and $\bar{D}^{0}$ mesons). While the correlation function in the first scenario is completely flat, the correlation function in the second scenario shows some tendency similar as in the experimental data. The increase at small $\Delta \varphi$ for the SPS $g g \rightarrow D^{0} D^{0}$ contribution is due to s-channel pole in the amplitude for $R R \rightarrow g g$ which we regularize by $\hat{s}>4 m_{c}^{2}$ condition. In the $k_{t}$-factorization, initial partons have transverse momenta, but final gluons may have equal rapidities even when $\Delta \varphi$ is far from $\pi$. 

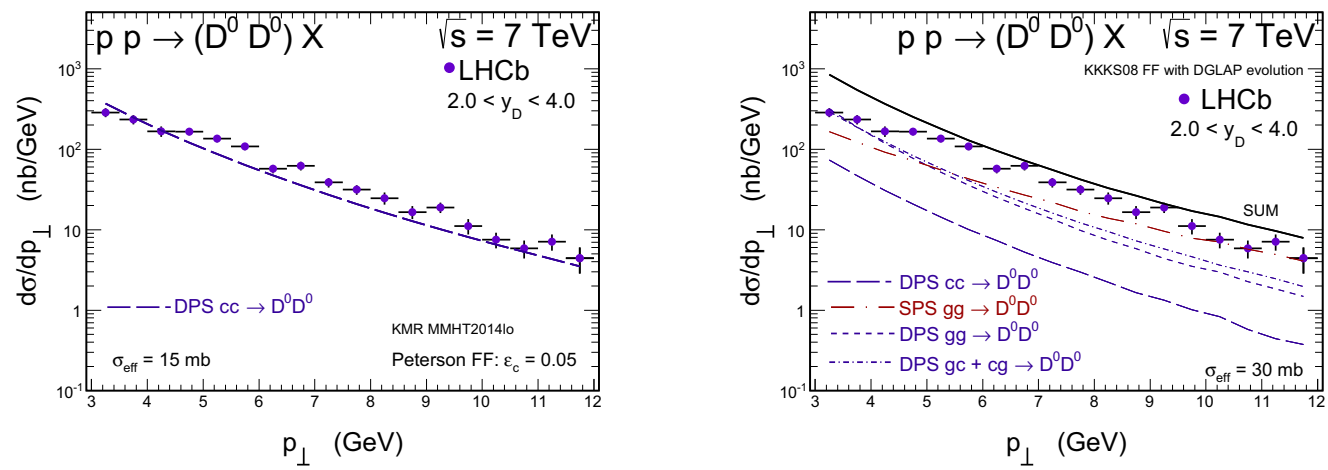

Figure 1. $D^{0}$ meson transverse momentum distribution within the LHCb acceptance region. The left panel is for the first scenario and for the Peterson $c \rightarrow D$ fragmentation function while the right panel is for the second scenario and for the fragmentation function that undergo DGLAP evolution equation.
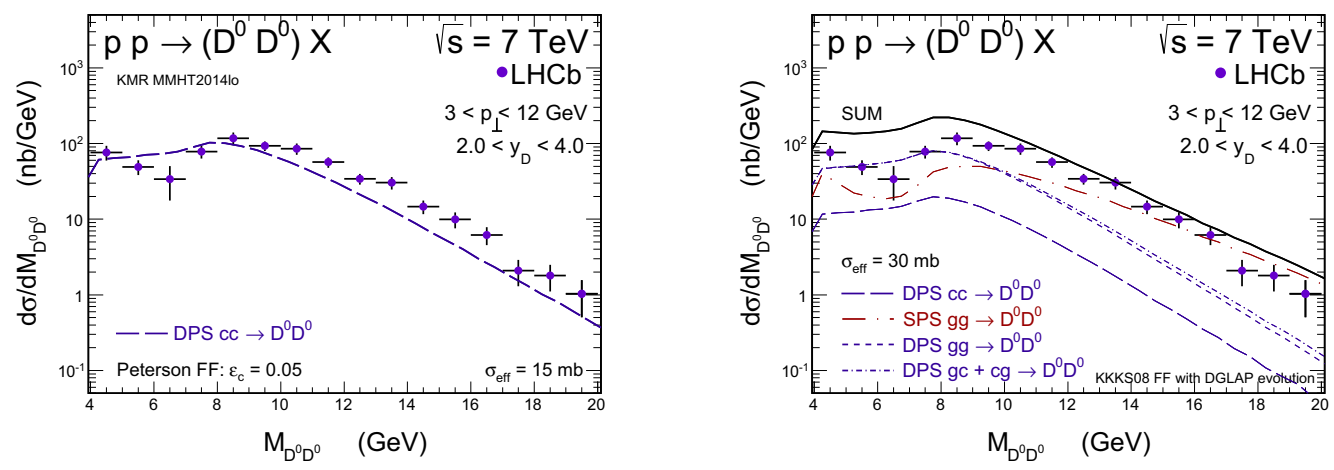

Figure 2. $M_{D^{0} D^{0}}$ dimeson invariant mass distribution within the LHCb acceptance region. The left panel is for the first scenario and for the Peterson $c \rightarrow D$ fragmentation function while the right panel is for the second scenario and for the fragmentation function that undergo DGLAP evolution equation.

\section{Conclusions}

The new multi-component scenario for double $D$ meson production give similar result as the first scenario with one subprocess $(c c \rightarrow D D)$ and fixed (scale-independent) fragmentation function. However, correlation observables, such as dimeson invariant mass or azimuthal correlations between $D$ mesons, are slightly better described in the second scenario as long as we consider only their shapes. To get the proper normalization of the cross sections calculated within the second scenario a much larger value of $\sigma_{\text {eff }}$ is needed.

The observed overestimation of the correlation observables in the second scenario comes from the region of small transverse momenta. It may be related to the fact that the fragmentation function used in the new scenario were obtained in the DGLAP formalism with massless $c$ quarks and $\bar{c}$ antiquarks which may be a too severe approximation, especially for low factorization scales (i.e. low trans- 
verse momenta) for fragmentation functions. On the other hand, the situation can be also improved when a proper transverse momentum dependence of $\sigma_{\text {eff }}$ and/or when perturbative-parton-splitting mechanisms will be included, but this needs further studies.
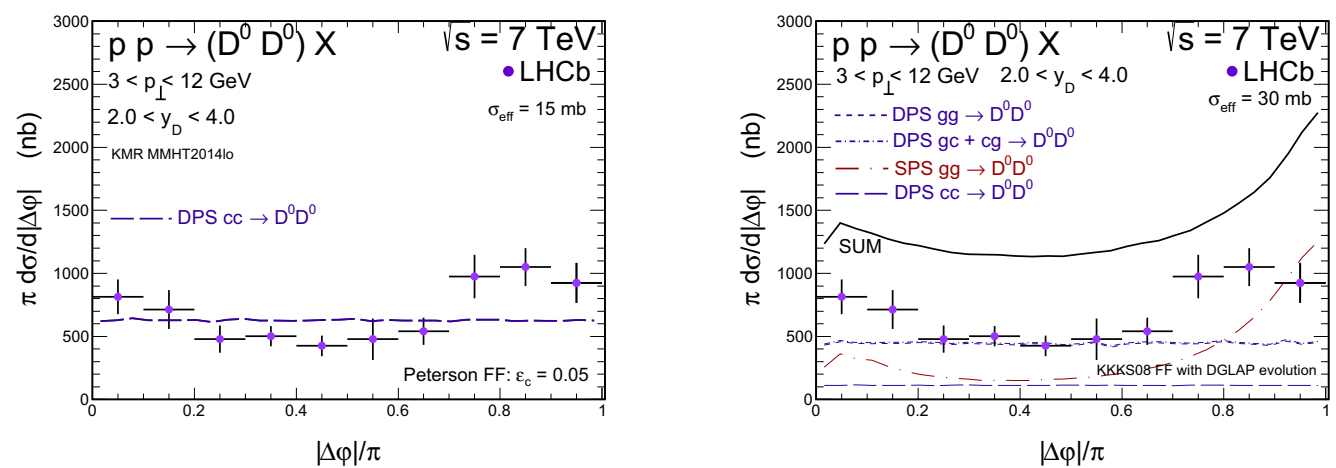

Figure 3. Distribution in azimuthal angle $\varphi_{D^{0} D^{0}}$ between the two $D^{0}$ mesons within the LHCb acceptance region. The left panel is for the first scenario and for the Peterson $c \rightarrow D$ fragmentation function while the right panel is for the second scenario and for the fragmentation function that undergo DGLAP evolution equation.

We thank V. A. Saleev and A. V. Shipilova for collaboration in obtaining results presented here.

\section{References}

[1] M. Łuszczak, R. Maciuła and A. Szczurek, Phys. Rev. D 85, 094034 (2012)

[2] R. Maciuła and A. Szczurek, Phys. Rev. D 87, 074039 (2013)

[3] A. van Hameren, R. Maciuła and A. Szczurek, Phys. Rev. D 89, 094019 (2014)

[4] R. Aaij et al. [LHCb Collaboration], J. High Energy Phys. 06, 141 (2012); [03, 108 (2014)]

[5] A. van Hameren, R. Maciuła and A. Szczurek, Phys. Lett. B 748, 167 (2015)

[6] C. Peterson, D. Schlatter, I. Schmitt and P. M. Zerwas, Phys. Rev. D 27, 105 (1983)

[7] B. A. Kniehl and G. Kramer, Phys. Rev. D 71, 094013 (2005)

[8] B. A. Kniehl and G. Kramer, Phys. Rev. D 74, 037502 (2006)

[9] R. Maciula and A. Szczurek, Phys. Rev. D 87, 094022 (2013)

[10] A. Karpishkov, M. Nefedov, V. A. Saleev and A. V. Shipilova, Phys. Rev. D 91, 054009 (2015)

[11] M. A. Nefedov, V. A. Saleev and A. V. Shipilova, Phys. Rev. D 87, 094030 (2013)

[12] T. Kneesch, B. A. Kniehl, G. Kramer and I. Schienbein, Nucl. Phys. B 799, 34 (2008)

[13] KKKS08-package: http://lapth.cnrs.fr/ffgenerator/

[14] R. Maciuła, V. A. Saleev, A. V. Shipilova and A. Szczurek, Phys. Lett. B 758, 458 (2016)

[15] J. R. Gaunt, R. Maciula and A. Szczurek, Phys. Rev. D 90, 054017 (2014) 